\title{
Reproductive Behaviour of Lemon (Citrus limon Burm.) Affected by Different Pruning Intensities and Integrated Nutrient Management under Various Growing Season
}

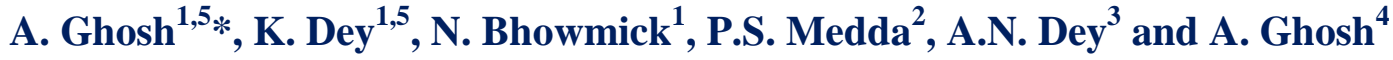 \\ ${ }^{1}$ Department of Pomology and Post Harvest Technology, Uttar Banga Krishi Viswavidyalaya, \\ Pundibari, Cooch Behar-736165, India \\ ${ }^{2}$ Department of Plantation Crops and Processing, Uttar Banga Krishi Viswavidyalaya, Pundibari, \\ Cooch Behar-736165, India \\ ${ }^{3}$ Department of Forestry, Uttar Banga Krishi Viswavidyalaya, Pundibari, Cooch Behar-736165 \\ ${ }^{4}$ Department of Agricultural Statictics, Uttar Banga Krishi Viswavidyalaya, Pundibari, \\ Cooch Behar-736165, India \\ ${ }^{5}$ Department of Fruits and Orchard Management, Bidhan Chandra Krishi Viswavidyalaya, \\ Mohanpur, Nadia-741252, India \\ *Corresponding author
}

\section{A B S T R A C T}

\begin{tabular}{|l|}
\hline Ke y w o r d s \\
Lemon, Nutrient \\
management, \\
Pruning, \\
Reproductive \\
behaviour.
\end{tabular}

The main objective of this study was to know the reproductive behaviour of lemon (Citrus limon Burm.) affected by different pruning intensities and integrated nutrient management under various growing season. The experiment was laid out in two factorial Randomized Block Design with four levels of pruning, seven levels of nutrient, consisting recommended dose of fertilizers (RDF) and different combinations of organic manure (Vermicompost), inorganic fertilizer, bio-fertilizer (Azotobacter), mycorrhiza (VAM) and their interaction to study their effect on plant reproductive behaviour during 2013 to 2015 on 9 year old lemon plants in three growing seasons. The investigation revealed that the reproductive parameters viz. number of flowers per plant, fruit set percentage and fruit yield were found highest in lightly pruned plants feed with $75 \%$ RDF + Vermicompost + Azotobacter + Vesicular Arbuscular Mycorrhiza at Ambe, Mrig and Hasth bahar respectively. Among the three season of cropping Ambe bahar recorded the best result in respect to yield followed by Mrig and Hasth bahar.

\section{Introduction}

Citrus is the most economically important fruit crop in the world, is grown in developed and developing countries and certainly constitutes one of the main sources of vitamin C. There is also an increasing demand of "high quality fresh citrus" driven by World Health Organization recommendations
(Iglesias et al., 2007). Assam Lemon is one of the important varieties of lemon, extensively grown in the north-eastern parts of India. In northern parts of West Bengal, it is early bearing with three fruiting season, viz. AprilMay, August-September and NovemberDecember. The earlier vegetative flushes of 
the previous season growth generally are more productive (Singh and Saxena, 2008). It was observed that the main reason for declining the productivity of the plant is unbalanced overcrowded orchard which also resulted in high disease-pest infestation (Singh and Dhaliwal, 2004). Therefore pruning is essential to maximize sunlight penetration which not only influences the flowering and fruit set but also enhances fruit quality and colour development. As lemon plants bears three times in a year, proper manuring and fertilization has also to be resorted for obtaining highest yields and quality production (Khehra and Bal, 2014). However, the continuous use of chemical fertilizers has degraded the soil health in terms of fertility, productivity and has also caused soil pollution. In such a situation, combine application of organic, inorganic and biofertilizers need to resort for avoiding the deleterious effect of chemical fertilizers and as well as improves physical properties of soil. As, information about the response of lemon against pruning and nutrient management is lacking for this area, the present investigation was conducted to know the reproductive behaviour of lemon (Citrus limon Burm.) affected by different pruning intensities and integrated nutrient management under various growing season.

\section{Materials and Methods}

The present investigation was carried out during 2013 to 2015 on 7 years old lemon cv. Assam lemon plants planted at $3 \mathrm{~m} \times 3 \mathrm{~m}$ spacing at Instructional farm of Uttar Banga Krishi Viswavidyalaya, Pundibari, Cooch Behar, West Bengal, India $\left(26^{0} 19^{\prime} 86^{\prime \prime} \mathrm{N}\right.$ latitude and $89^{\circ} 23^{\prime} 53^{\prime \prime} \mathrm{E}$ longitude). There were 4 levels of pruning, namely $\mathrm{P} 0-\mathrm{No}$ pruning (Control), $\mathrm{P} 1-25 \mathrm{~cm}$ pruning from the terminal portion of the shoot, P2- $50 \mathrm{~cm}$ pruning from the terminal portion of the shoot, $\mathrm{P} 3-75 \mathrm{~cm}$ pruning from the terminal portion of the shoot and 7 treatments of nutrient management viz. N1- $100 \%$ Recommended Dose of Fertilizer (N@210g/plant- P@140g/plant- K@210g/ plant), N2- Vermicompost (20kg/plant) + Azotobacter (18g/plant) +Vesicular Arbuscular Mycorrhiza (150g/plant), N3Vermicompost, N4- $\quad 75 \%$ RDF+Vermicompost +Azotobacter + Vesicular Arbuscular Mycorrhiza, N5- 75\% RDF+Vermicompost, N6- 50\% RDF+ Vermicompost + Azotobacter + Vesicular Arbuscular Mycorrhiza and N7- 50\% RDF+Vermicompost were applied alone and in combination with different levels of the pruning. The experiment was laid out in two factorial asymmetrical randomized block design (RBD) and 28 treatment combination (4 levels of pruning and 7 levels of nutrient) with 3 replications and 6 plants were kept in each treatment. All levels of pruning were done on $21^{\text {st }}$ November, 2013, after harvesting of Mrigbahar. Nitrogenous fertilizer was applied in two split doses. Firstly, half dose of nitrogen and full dose of phosphorus, potassium and vermicompost were applied in February, 2014 and rest half of nitrogen was applied in April, 2014. Azotobacter and Vesicular Arbuscular Mycorrhiza were applied in December, 2013, after harvesting of Mrigbahar. All the reproductive parameters viz. number of flowers per plant, fruit set $(\%)$, fruit retention (\%) and fruit yield were recorded from six tagged plants for each treatment at three distinct seasons viz. Ambe, Mrig and Hasth bahar respectively. Analysis of variance (one way classified data) for each parameter was performed using ProcGlm of Statistical Analysis System (SAS) software (version 9.3). Mean separation for different treatment under different parameter were performed using Least Significant Different (LSD) test $(\mathrm{P} \leq 0.05)$. Normality of residuals under the assumsion of ANOVA was tested using Kolmogrov-Smirnov, Shapiro-Wilk, Cramer- 
Von Mises and Anderson Darling procedure using Proc-Univariate procedure of (version 9.3) SAS (Gomez and Gomez, 1983).

\section{Results and Discussion}

\section{Number of flower}

Experimental results on number of flowers per plant were showed significant variation in all the season under different pruning and nutrient level (Table 1). Maximum number of flowers per plant (408.33, 378.33 and 259) were recorded in P1 $(25 \mathrm{~cm}$ pruning from the terminal portion of the shoot) at Ambe, Mrig and Hasth bahar followed by unpruned plantsP0 (388.33, 363.33 and 245) at Ambe, Mrig and Hasth bahar. The minimum number of flowers per plant was observed in P3 (75 $\mathrm{cm}$ pruning from the terminal portion of the shoot) (288, 252 and 177.33) at Ambe, Mrig and Hasth bahar respectively. The significantly highest number of flowers per plant was recorded (399, 371.67 and 250.33) in $\mathrm{N} 4 \quad(75 \% \mathrm{RDF}+$ Vermicompost + Azotobacter + Vesicular Arbuscular Mycorrhiza) and the lowest number (386.33, 360 and 224.33) were observed in N3 (Vermicompost) at three seasons respectively. The interaction between different pruning and nutrient level (Table 2) was statistically at par with respect to number of flowers proved that treatment combination have no effect on this parameter. Data revealed that $\mathrm{T}_{11}\left(\mathrm{P}_{1} \mathrm{~N}_{4}\right)$ gave the maximum number of flowers per plant (454.33, 424.67 and 320) at Ambe, Mrig and Hasth bahar followed by $\mathrm{T}_{13}\left(\mathrm{P}_{1} \mathrm{~N}_{6}\right)(446,413$ and 290.33), whereas these were minimum (242.33, 205 and 107.67) in $\mathrm{T}_{24}\left(\mathrm{P}_{3} \mathrm{~N}_{3}\right)$ at three seasons respectively. The lowest number of flowers in severely pruned plants was due to loss of potential bearing wood of these plants (Nath and Baruah, 1999). The role of biofertilizer in fixation of atmospheric nitrogen and VAM involved in solubilization of phosphate are responsible for maintaining better soil environment which ultimately reflected in the flowering of the tree (Yadav et al., 2011). Similar findings also reported in bael (Singh et al., 2009).

\section{Fruit set (\%)}

The data pertaining to fruit set $(\%)$ have been revealed that all the data were significantly different under different pruning and nutrient treatments. Maximum fruit set (\%) was recorded (Table 1) in P1 $(25 \mathrm{~cm}$ pruning from the terminal portion of the shoot) at Ambe, Mrig and Hasth bahar $(63.43 \%, 52.22 \%$ and $40.93 \%$ ) and the minimum was observed in (P0) unpruned plants $(52.53 \%, 32.20 \%$ and $23.27 \%$ ) at Ambe, Mrig and Hasth bahar respectively. In case of nutrients, the highest fruit set $(\%)$ was recorded $(54.14 \%, 38.21 \%$ and $31.16 \%)$ in $\mathrm{N} 4 \quad(75 \% \quad \mathrm{RDF}+$ Vermicompost + Azotobacter + Vesicular Arbuscular Mycorrhiza) at Ambe, Mrig and Hasth bahar respectively. The interaction effect (Table 2) between pruning and nutrient was highly significant with respect to fruit set $(\%)$ at Ambe, Mrig and Hasth bahar. It revealed that $\mathrm{T}_{11}\left(\mathrm{P}_{1} \mathrm{~N}_{4}\right)$ gave the maximum fruit set $(\%)(69.11 \%, 52.51 \%$ and $48.75 \%)$ at Ambe, Mrig and Hasth bahar followed by $\mathrm{T}_{13}$ $\left(\mathrm{P}_{1} \mathrm{~N}_{6}\right) \quad(68.83 \%, \quad 52.06 \%$ and $47.19 \%)$, whereas fruit set $(\%)$ was minimum $(51 \%$, $31.94 \%$ and $21.84 \%)$ in $\mathrm{T}_{3}\left(\mathrm{P}_{0} \mathrm{~N}_{3}\right)$ at Ambe, Mrig and Hasth bahar respectively. These results are in close conformity with the findings in guava (Shaban and Haseeb, 2009). Increase in fruit set (\%) might be due to the optimum supply of nutrients in integrated way which resulted higher photosynthates production and thereby enhanced fruit set (Yadav et al., 2011). 
Table.1 Effect of pruning and nutrient management on flowering and fruiting of lemon cv. Assam Lemon

\section{Ambe bahar}

\begin{tabular}{|c|c|c|c|c|c|c|c|c|c|}
\hline Treatments & $\begin{array}{l}\text { No. of } \\
\text { flowers/plant }\end{array}$ & $\begin{array}{l}\text { Fruit } \\
\text { set } \\
(\%)\end{array}$ & $\begin{array}{l}\text { Fruit } \\
\text { retention } \\
(\%)\end{array}$ & $\begin{array}{l}\text { No. of } \\
\text { flowers/plant }\end{array}$ & $\begin{array}{l}\text { Fruit } \\
\text { set } \\
(\%)\end{array}$ & $\begin{array}{l}\text { Fruit } \\
\text { retention } \\
(\%)\end{array}$ & $\begin{array}{l}\text { No. of } \\
\text { flowers/plant }\end{array}$ & $\begin{array}{l}\text { Fruit set } \\
(\%)\end{array}$ & $\begin{array}{l}\text { Fruit } \\
\text { retention } \\
(\%)\end{array}$ \\
\hline $\mathrm{P}_{0}$ & $388.33 d$ & $52.53 \mathrm{~d}$ & $\begin{array}{c}50.49 \\
(45.29) \mathrm{d}\end{array}$ & $363.33 \mathrm{~d}$ & $32.2 \mathrm{~d}$ & $39.32 \mathrm{~d}$ & $245 \mathrm{~d}$ & $\begin{array}{c}23.27 \\
(28.86) \mathrm{d} \\
\end{array}$ & $\begin{array}{c}21.05 \\
(27.35) \mathrm{d}\end{array}$ \\
\hline $\mathrm{P}_{1}$ & $408.33 \mathrm{c}$ & $63.43 \mathrm{c}$ & $\begin{array}{c}84.56 \\
(66.89) \mathrm{c} \\
\end{array}$ & $378.33 \mathrm{c}$ & $50.22 \mathrm{c}$ & $67.89 \mathrm{c}$ & $259 \mathrm{c}$ & $\begin{array}{c}40.93 \\
(39.76) \mathrm{c}\end{array}$ & $\begin{array}{c}36.79 \\
(37.35) \mathrm{c}\end{array}$ \\
\hline $\mathrm{P}_{2}$ & $349.67 \mathrm{~b}$ & $60.63 \mathrm{~b}$ & $\begin{array}{c}68.87 \\
(56.11) \mathrm{b} \\
\end{array}$ & $314.33 b$ & $45.81 \mathrm{~b}$ & $61.11 \mathrm{~b}$ & $212 b$ & $\begin{array}{c}37.74 \\
(37.88) \mathrm{b} \\
\end{array}$ & $\begin{array}{c}33.75 \\
(35.55) \mathrm{b} \\
\end{array}$ \\
\hline $\mathrm{P}_{3}$ & $288 \mathrm{a}$ & $58.33 \mathrm{a}$ & $\begin{array}{c}63.69 \\
(52.95) \mathrm{a} \\
\end{array}$ & $252 a$ & $41.67 \mathrm{a}$ & $56.19 a$ & $177.33 \mathrm{a}$ & $\begin{array}{c}32.14 \\
(34.51) \mathrm{a}\end{array}$ & $\begin{array}{c}28.07 \\
(32.01) \mathrm{a}\end{array}$ \\
\hline $\operatorname{SEm}( \pm)$ & 5.93 & 0.07 & 0.01 & 7.33 & 0.01 & 0.01 & 5.65 & 0.01 & 0.01 \\
\hline $\begin{array}{c}\text { LSD } \\
(\mathbf{P} \leq \mathbf{0 . 0 5})\end{array}$ & 16.82 & 0.20 & 0.03 & 20.78 & 0.02 & 0.02 & 16.02 & 0.02 & 0.02 \\
\hline $\mathrm{N}_{1}$ & $388.33 \mathrm{bc}$ & $52.53 \mathrm{c}$ & $\begin{array}{c}50.49 \\
(45.29) \mathrm{e}\end{array}$ & $363.33 \mathrm{~cd}$ & $32.2 \mathrm{e}$ & $39.32 \mathrm{e}$ & $245 b c$ & $\begin{array}{c}23.27 \\
(28.86) \mathrm{e}\end{array}$ & $\begin{array}{c}21.05 \\
(27.35) \mathrm{e}\end{array}$ \\
\hline $\mathrm{N}_{2}$ & $386.33 \mathrm{bc}$ & $51.25 \mathrm{~d}$ & $\begin{array}{c}48.48 \\
(44.14) \mathrm{f}\end{array}$ & $362.33 \mathrm{~d}$ & $32.02 \mathrm{f}$ & $33.62 \mathrm{f}$ & $245 \mathrm{~cd}$ & $\begin{array}{c}21.63 \\
(27.69) \mathrm{f}\end{array}$ & $\begin{array}{c}20.75 \\
(27.13) \mathrm{f}\end{array}$ \\
\hline $\mathrm{N}_{3}$ & $386.33 \mathrm{c}$ & $51 \mathrm{~d}$ & $\begin{array}{c}40.61 \\
(39.58) \mathrm{g}\end{array}$ & $360 \mathrm{~d}$ & $31.94 \mathrm{~g}$ & $31.3 \mathrm{~g}$ & $224.33 d$ & $\begin{array}{c}21.84 \\
(27.83) \mathrm{g}\end{array}$ & $\begin{array}{c}16.33 \\
(23.81) \mathrm{g}\end{array}$ \\
\hline $\mathrm{N}_{4}$ & $399 a$ & $54.14 \mathrm{a}$ & $\begin{array}{c}52.78 \\
(46.61) \mathrm{a}\end{array}$ & $371.67 \mathrm{a}$ & $38.21 \mathrm{a}$ & $42.25 \mathrm{a}$ & $250.33 \mathrm{a}$ & $\begin{array}{c}31.16 \\
(33.96) \mathrm{a}\end{array}$ & $\begin{array}{c}21.79 \\
(27.83) \mathrm{a}\end{array}$ \\
\hline $\mathrm{N}_{5}$ & $395.67 \mathrm{ab}$ & $53.07 \mathrm{~b}$ & $\begin{array}{c}52.38 \\
(46.38) \mathrm{c}\end{array}$ & $365.33 \mathrm{abc}$ & $33.67 \mathrm{c}$ & $41.46 \mathrm{c}$ & 245.33abc & $\begin{array}{c}30.57 \\
(33.58) \mathrm{c}\end{array}$ & $\begin{array}{c}21.33 \\
(27.49) \mathrm{c}\end{array}$ \\
\hline $\mathrm{N}_{6}$ & $397.67 \mathrm{a}$ & $54.06 \mathrm{a}$ & $\begin{array}{c}52.09 \\
(46.20) \mathrm{b}\end{array}$ & 371.33ab & $34.47 \mathrm{~b}$ & $42.19 b$ & $246.33 \mathrm{ab}$ & $\begin{array}{c}30.85 \\
(33.77) \mathrm{b}\end{array}$ & $\begin{array}{c}21.05 \\
(27.35) b\end{array}$ \\
\hline $\mathrm{N}_{7}$ & $391 b c$ & $52.94 \mathrm{~b}$ & $\begin{array}{c}51.69 \\
(45.97) \mathrm{d}\end{array}$ & $364 \mathrm{bcd}$ & $32.42 \mathrm{~d}$ & $40.68 \mathrm{~d}$ & $245 b c$ & $\begin{array}{c}28.16 \\
(32.08) \mathrm{d}\end{array}$ & $\begin{array}{c}20.29 \\
(26.78) \mathrm{d}\end{array}$ \\
\hline $\operatorname{SEm}( \pm)$ & 7.85 & 0.09 & 0.01 & 9.69 & 0.01 & 0.01 & 7.47 & 0.01 & 0.01 \\
\hline $\begin{array}{l}\text { LSD } \\
(P \leq 0.05)\end{array}$ & 22.25 & 0.27 & 0.03 & 27.49 & 0.03 & 0.03 & 21.19 & 0.03 & 0.03 \\
\hline
\end{tabular}


Table.2 Interaction effect of pruning and nutrient management on flowering and fruiting of lemon cv. Assam Lemon

\begin{tabular}{|c|c|c|c|c|c|c|c|c|c|}
\hline & Ambe bahar & & & Mrig bahar & & & Hasth bahar & & \\
\hline Treatments & $\begin{array}{l}\text { No. of } \\
\text { flowers/plant }\end{array}$ & $\begin{array}{l}\text { Fruit } \\
\text { set } \\
(\%) \\
\end{array}$ & $\begin{array}{l}\text { Fruit } \\
\text { retention } \\
(\%)\end{array}$ & $\begin{array}{l}\text { No. of } \\
\text { flowers/plant }\end{array}$ & $\begin{array}{l}\text { Fruit } \\
\text { set } \\
(\%) \\
\end{array}$ & $\begin{array}{l}\text { Fruit } \\
\text { retention } \\
(\%)\end{array}$ & $\begin{array}{l}\text { No. of } \\
\text { flowers/plant }\end{array}$ & Fruit set (\%) & $\begin{array}{l}\text { Fruit } \\
\text { retention }(\%)\end{array}$ \\
\hline $\mathrm{T}_{1}\left(\mathrm{P}_{0} \mathrm{~N}_{1}\right)$ & 388.33 & 52.53 & $50.49(45.29)$ & 363.33 & 32.2 & 39.32 & 245 & $23.27(28.86)$ & $21.05(27.35)$ \\
\hline $\mathrm{T}_{2}\left(\mathrm{P}_{0} \mathrm{~N}_{2}\right)$ & 386.33 & 51.25 & $48.48(44.14)$ & 362.33 & 32.02 & 33.62 & 245 & $21.63(27.69)$ & $20.75(27.13)$ \\
\hline $\mathrm{T}_{3}\left(\mathrm{P}_{0} \mathrm{~N}_{3}\right)$ & 386.33 & 51 & $40.61(39.58)$ & 360 & 31.94 & 31.3 & 224.33 & $21.84(27.83)$ & $16.33(23.81)$ \\
\hline $\mathrm{T}_{4}\left(\mathrm{P}_{0} \mathrm{~N}_{4}\right)$ & 399 & 54.14 & $52.78(46.61)$ & 371.67 & 38.21 & 42.25 & 250.33 & $31.16(33.96)$ & $21.79(27.83)$ \\
\hline $\mathrm{T}_{6}\left(\mathrm{P}_{0} \mathrm{~N}_{6}\right)$ & 397.67 & 54.06 & $52.09(46.20)$ & 371.33 & 34.47 & 42.19 & 246.33 & $30.85(33.77)$ & $21.05(27.35)$ \\
\hline $\mathrm{T}_{7}\left(\mathrm{P}_{0} \mathrm{~N}_{7}\right)$ & 391 & 52.94 & $51.69(45.97)$ & 364 & 32.42 & 40.68 & 245 & $28.16(32.08)$ & $20.29(26.78)$ \\
\hline $\mathrm{T}_{8}\left(\mathrm{P}_{1} \mathrm{~N}_{1}\right)$ & 408.33 & 63.43 & $84.56(66.89)$ & 378.33 & 50.22 & 67.89 & 259 & $40.93(39.76)$ & $36.79(37.350$ \\
\hline $\mathrm{T}_{9}\left(\mathrm{P}_{1} \mathrm{~N}_{2}\right)$ & 405.67 & 63.11 & $84.38(66.74)$ & 375.67 & 48.71 & 67.76 & 255 & $40.00(39.23)$ & $35.29(36.45)$ \\
\hline $\mathrm{T}_{10}\left(\mathrm{P}_{1} \mathrm{~N}_{3}\right)$ & 403.67 & 62.92 & $83.07(65.73)$ & 375 & 48.53 & 67.03 & 252 & $39.29(38.82)$ & $34.34(35.85)$ \\
\hline $\mathrm{T}_{11}\left(\mathrm{P}_{1} \mathrm{~N}_{4}\right)$ & 454.33 & 69.11 & $90.76(72.34)$ & 424.67 & 52.51 & 70.85 & 320 & $48.75(44.51)$ & $40.38(39.47)$ \\
\hline $\mathrm{T}_{12}\left(\mathrm{P}_{1} \mathrm{~N}_{5}\right)$ & 416.67 & 67.2 & $85.71(67.78)$ & 389.33 & 51.37 & 69.5 & 276 & $43.12(41.03)$ & $39.5(38.94)$ \\
\hline $\mathrm{T}_{14}\left(\mathrm{P}_{1} \mathrm{~N}_{7}\right)$ & 412.67 & 65.43 & $85.56(67.70)$ & 379.67 & 51.1 & 68.56 & 265.67 & $42.53(40.69)$ & $38.05(38.12)$ \\
\hline $\mathrm{T}_{15}\left(\mathrm{P}_{2} \mathrm{~N}_{1}\right)$ & 349.67 & 60.63 & $68.87(56.11)$ & 314.33 & 45.81 & 61.11 & 212 & $37.74(37.88)$ & $33.75(35.55)$ \\
\hline $\mathrm{T}_{16}\left(\mathrm{P}_{2} \mathrm{~N}_{2}\right)$ & 346.67 & 59.71 & $64.73(53.55)$ & 313 & 45.37 & 59.15 & 211.67 & $37.32(37.64)$ & $32.91(35)$ \\
\hline $\mathrm{T}_{17}\left(\mathrm{P}_{2} \mathrm{~N}_{3}\right)$ & 337.33 & 59.59 & $64.68(53.55)$ & 306 & 44.44 & 58.82 & 210.67 & $35.13(36.33)$ & $32.43(34.70)$ \\
\hline $\mathrm{T}_{18}\left(\mathrm{P}_{2} \mathrm{~N}_{4}\right)$ & 380 & 62.63 & $82.77(65.50)$ & 358 & 48.04 & 63.37 & 223 & $39.01(38.65)$ & $34.48(35.97)$ \\
\hline $\mathrm{T}_{19}\left(\mathrm{P}_{2} \mathrm{~N}_{5}\right)$ & 373 & 62.47 & $73.39(58.95)$ & 352 & 47.73 & 62.5 & 222 & $38.29(38.23)$ & $34.12(35.73)$ \\
\hline $\mathrm{T}_{20}\left(\mathrm{P}_{2} \mathrm{~N}_{6}\right)$ & 378 & 62.7 & $74.68(59.80)$ & 353.67 & 48.07 & 62.94 & 223 & $38.57(38.41)$ & $33.72(35.49)$ \\
\hline $\mathrm{T}_{21}\left(\mathrm{P}_{2} \mathrm{~N}_{7}\right)$ & 351 & 62.39 & $70.78(57.29)$ & 320 & 46.25 & 61.49 & 216.67 & $37.85(38)$ & $32.93(35)$ \\
\hline $\mathrm{T}_{22}\left(\mathrm{P}_{3} \mathrm{~N}_{1}\right)$ & 288 & 58.33 & $63.69(52.95)$ & 252 & 41.67 & 56.19 & 177.33 & $32.14(34.51)$ & $28.07(32.01)$ \\
\hline $\mathrm{T}_{23}\left(\mathrm{P}_{3} \mathrm{~N}_{2}\right)$ & 280 & 54.64 & $63.4(52.77)$ & 212.67 & 41.38 & 55.68 & 163.33 & $31.84(34.33)$ & $26.92(31.24)$ \\
\hline $\mathrm{T}_{24}\left(\mathrm{P}_{3} \mathrm{~N}_{3}\right)$ & 242.33 & 54.47 & $62.88(52.48)$ & 205 & 40.98 & 54.76 & 107.67 & $31.58(34.20)$ & $26.47(30.98)$ \\
\hline $\mathrm{T}_{25}\left(\mathrm{P}_{3} \mathrm{~N}_{4}\right)$ & 327.67 & 59.51 & $64.62(53.49)$ & 296 & 44.26 & 58.78 & 209.33 & $34.4(35.91)$ & $31.94(34.39)$ \\
\hline $\mathrm{T}_{26}\left(\mathrm{P}_{3} \mathrm{~N}_{5}\right)$ & 295.67 & 58.85 & $64.37(53.37)$ & 276.67 & 41.93 & 56.03 & 199.33 & $33.61(35.43)$ & $29.85(33.15)$ \\
\hline $\mathrm{T}_{27}\left(\mathrm{P}_{3} \mathrm{~N}_{6}\right)$ & 325.67 & 58.96 & $64.58(53.49)$ & 285.67 & 42.36 & 57.85 & 209 & $33.97(35.67)$ & $30.99(33.83)$ \\
\hline $\mathrm{T}_{28}\left(\mathrm{P}_{3} \mathrm{~N}_{7}\right)$ & 294.67 & 58.71 & $64.16(53.25)$ & 254.33 & 41.68 & 55.66 & 182.67 & $32.3(34.63)$ & $28.81(32.46)$ \\
\hline $\operatorname{SEm}( \pm)$ & 15.70 & 0.19 & 0.02 & 19.39 & 0.02 & 0.02 & 14.95 & 0.02 & 0.02 \\
\hline $\mathrm{LSD}(\mathrm{P} \leq \mathbf{0 . 0 5})$ & NS & 0.54 & 0.07 & NS & 0.06 & 0.05 & NS & 0.06 & 0.05 \\
\hline
\end{tabular}


Table.3 Effect of pruning and nutrient management on yield of lemon cv. Assam Lemon

\begin{tabular}{|c|c|c|c|c|c|c|}
\hline & $\begin{array}{l}\text { Ambe } \\
\text { bahar }\end{array}$ & & $\begin{array}{l}\text { Mrig } \\
\text { bahar }\end{array}$ & & $\begin{array}{l}\text { Hasth } \\
\text { bahar }\end{array}$ & \\
\hline Treatments & $\begin{array}{l}\text { Total } \\
\text { number } \\
\text { of } \\
\text { Harvested } \\
\text { fruits }\end{array}$ & $\begin{array}{l}\text { Fruit } \\
\text { yield } \\
\text { (kg/plant) }\end{array}$ & $\begin{array}{l}\text { Total } \\
\text { number } \\
\text { of } \\
\text { Harvested } \\
\text { fruits }\end{array}$ & $\begin{array}{l}\text { Fruit } \\
\text { yield } \\
\text { (kg/plant) }\end{array}$ & $\begin{array}{l}\text { Total } \\
\text { number } \\
\text { of } \\
\text { Harvested } \\
\text { fruits }\end{array}$ & $\begin{array}{l}\text { Fruit } \\
\text { yield } \\
\text { (kg/plant) }\end{array}$ \\
\hline $\mathrm{P}_{0}$ & $103 d$ & $11.52 \mathrm{~d}$ & $46 d$ & $5.48 \mathrm{~d}$ & $12 d$ & $1.38 \mathrm{~d}$ \\
\hline $\mathrm{P}_{1}$ & $219 \mathrm{c}$ & $26.71 \mathrm{c}$ & $129 \mathrm{c}$ & $16.86 \mathrm{c}$ & $39 \mathrm{c}$ & $4.96 \mathrm{c}$ \\
\hline $\mathrm{P}_{2}$ & $146 \mathrm{~b}$ & $18.11 \mathrm{~b}$ & $88 \mathrm{~b}$ & $11.80 \mathrm{~b}$ & $27 b$ & $3.45 b$ \\
\hline $\mathrm{P}_{3}$ & $107 \mathrm{a}$ & $15.06 \mathrm{a}$ & $59 a$ & $9.13 \mathrm{a}$ & $16 \mathrm{a}$ & $2.36 \mathrm{a}$ \\
\hline SEm ( \pm$)$ & 1.13 & 0.19 & 0.93 & 0.14 & 0.89 & 0.12 \\
\hline $\operatorname{LSD}(\mathbf{P} \leq 0.05)$ & 3.20 & 0.54 & 2.63 & 0.40 & 2.52 & 0.34 \\
\hline $\mathrm{N}_{1}$ & $103 \mathrm{e}$ & $11.52 \mathrm{e}$ & $46 \mathrm{~d}$ & $5.48 \mathrm{~d}$ & $12 \mathrm{de}$ & $1.38 \mathrm{de}$ \\
\hline $\mathrm{N}_{2}$ & $96 \mathrm{f}$ & $10.72 \mathrm{f}$ & $39 \mathrm{e}$ & $4.65 \mathrm{e}$ & $11 \mathrm{ef}$ & $1.27 \mathrm{ef}$ \\
\hline $\mathrm{N}_{3}$ & $80 \mathrm{~g}$ & $8.86 \mathrm{~g}$ & $36 \mathrm{e}$ & $4.25 \mathrm{e}$ & $8 f$ & $0.92 \mathrm{f}$ \\
\hline $\mathrm{N}_{4}$ & $114 \mathrm{a}$ & $13.83 \mathrm{a}$ & $60 \mathrm{a}$ & $7.67 \mathrm{a}$ & $17 \mathrm{a}$ & $2.14 \mathrm{a}$ \\
\hline $\mathrm{N}_{5}$ & $110 \mathrm{c}$ & $13.25 \mathrm{c}$ & $51 \mathrm{c}$ & $6.51 \mathrm{c}$ & $16 \mathrm{bc}$ & $2 b c$ \\
\hline $\mathrm{N}_{6}$ & $112 b$ & $13.59 \mathrm{~b}$ & $54 b$ & $6.89 b$ & $16 \mathrm{ab}$ & $2.01 \mathrm{ab}$ \\
\hline $\mathrm{N}_{7}$ & $107 d$ & $12.14 \mathrm{~d}$ & $48 \mathrm{~d}$ & $5.77 \mathrm{~d}$ & $14 \mathrm{~cd}$ & $1.63 \mathrm{~cd}$ \\
\hline $\operatorname{SEm}( \pm)$ & 1.49 & 0.25 & 1.23 & 0.19 & 1.17 & 0.16 \\
\hline $\mathrm{LSD}(\mathrm{P}<0.05)$ & 4.23 & 0.71 & 3.48 & $\mathbf{0 . 5 3}$ & $\mathbf{3 . 3 3}$ & 0.45 \\
\hline
\end{tabular}

\section{Fruit retention (\%)}

Observations on fruit retention (\%) under different treatments and their combination have been presented in table 1 were statistically significant in three cropping seasons. The significantly highest fruit retention (\%) was recorded in P1 $(25 \mathrm{~cm}$ pruning from the terminal portion of the shoot) at Ambe, Mrig and Hasth bahar $(84.56 \%, 67.89 \%$ and $36.79 \%)$ and the lowest retention (\%) was observed in unpruned plants $(50.49 \%, 39.32 \%$ and $21.05 \%)$ at Ambe, Mrig and Hasth bahar respectively. The significantly highest fruit retention (\%) was recorded $(52.78 \%, 42.25 \%$ and $21.79 \%)$ in $\mathrm{N} 4 \quad(75 \%$ RDF+ Vermicompost + Azotobacter + Vesicular Arbuscular Mycorrhiza) at Ambe, Mrig and Hasth bahar. The interaction effect between pruning and nutrient revealed (Table 2) that $\mathrm{T}_{11}\left(\mathrm{P}_{1} \mathrm{~N}_{4}\right)$ gave the significantly maximum fruit retention (\%) $(90.76 \%, 70.85 \%$ and $40.38 \%)$ at Ambe, Mrig and Hasth bahar followed by
$\mathrm{T}_{13}\left(\mathrm{P}_{1} \mathrm{~N}_{6}\right)(89.90 \%, 69.77 \%$ and $40.15 \%)$, whereas minimum fruit retention (\%) was recorded $(40.61 \%, 31.30 \%$ and $16.33 \%)$ in $\mathrm{T}_{3}$ $\left(\mathrm{P}_{0} \mathrm{~N}_{3}\right)$ at three seasons. It might be due to combination use of organic and inorganic fertilizers and better nutrient availability from them which was enhanced by biofertilizer and vesicular arbuscular mycorrhiza resulted in better more retention of fruits at harvest. Fruit yield was significantly different in all seasons under different pruning and nutrient treatments.

\section{Fruit yield}

The data pertaining to fruit yield revealed that maximum number of harvested fruits was recorded (Tables 3 and 4) in P1 (26.71 $\mathrm{kg} /$ plant, $16.86 \mathrm{~kg} /$ plant and $4.96 \mathrm{~kg} / \mathrm{plant})$ followed by P2 (18.11 kg/plant, 11.80 $\mathrm{kg} /$ plant and $3.45 \mathrm{~kg} / \mathrm{plant}$ ) and the lowest result was found in (P0) unpruned plants $(1.52 \mathrm{~kg} /$ plant, $5.48 \mathrm{~kg} / \mathrm{plant}$ and 1.38 $\mathrm{kg} /$ plant) at Ambe, Mrig and Hasth bahar 
respectively. The significantly highest fruit yield was recorded $(13.83 \mathrm{~kg} / \mathrm{plant}, 7.67$ $\mathrm{kg} /$ plant $2.14 \mathrm{~kg} / \mathrm{plant})$ in $\mathrm{N} 4(75 \% \mathrm{RDF}+$
Vermicompost + Azotobacter + Vesicular Arbuscular Mycorrhiza) at Ambe, Mrig and Hasth bahar.

Table.4 Interaction effect of pruning and nutrient management on yield of lemon cv. Assam Lemon

\begin{tabular}{|c|c|c|c|c|c|c|}
\hline & Ambe bahar & & Mrig bahar & & Hasth bahar & \\
\hline Treatments & $\begin{array}{l}\text { Total } \\
\text { number of } \\
\text { Harvested } \\
\text { fruits }\end{array}$ & $\begin{array}{l}\text { Fruit } \\
\text { yield } \\
\text { (kg/plant) }\end{array}$ & $\begin{array}{l}\text { Total } \\
\text { number of } \\
\text { Harvested } \\
\text { fruits }\end{array}$ & $\begin{array}{l}\text { Fruit } \\
\text { yield } \\
\text { (kg/plant) }\end{array}$ & $\begin{array}{l}\text { Total number } \\
\text { of Harvested } \\
\text { fruits }\end{array}$ & $\begin{array}{l}\text { Fruit } \\
\text { yield } \\
\text { (kg/plant) }\end{array}$ \\
\hline $\mathrm{T}_{1}\left(\mathrm{P}_{0} \mathrm{~N}_{1}\right)$ & 103 & 11.52 & 46 & 5.48 & 12 & 1.38 \\
\hline $\mathrm{T}_{2}\left(\mathrm{P}_{0} \mathrm{~N}_{2}\right)$ & 96 & 10.72 & 39 & 4.65 & 11 & 1.27 \\
\hline $\mathrm{T}_{3}\left(\mathrm{P}_{0} \mathrm{~N}_{3}\right)$ & 80 & 8.86 & 36 & 4.25 & 8 & 0.92 \\
\hline $\mathrm{T}_{4}\left(\mathrm{P}_{0} \mathrm{~N}_{4}\right)$ & 114 & 13.83 & 60 & 7.67 & 17 & 2.14 \\
\hline $\mathrm{T}_{5}\left(\mathrm{P}_{0} \mathrm{~N}_{5}\right)$ & 110 & 13.25 & 51 & 6.51 & 16 & 2 \\
\hline $\mathrm{T}_{6}\left(\mathrm{P}_{0} \mathrm{~N}_{6}\right)$ & 112 & 13.59 & 54 & 6.89 & 16 & 2.01 \\
\hline $\mathrm{T}_{7}\left(\mathrm{P}_{0} \mathrm{~N}_{7}\right)$ & 107 & 12.14 & 48 & 5.77 & 14 & 1.63 \\
\hline $\mathrm{T}_{8}\left(\mathrm{P}_{1} \mathrm{~N}_{1}\right)$ & 219 & 26.71 & 129 & 16.86 & 39 & 4.96 \\
\hline $\mathrm{T}_{9}\left(\mathrm{P}_{1} \mathrm{~N}_{2}\right)$ & 216 & 26.28 & 124 & 16.17 & 36 & 4.56 \\
\hline $\mathrm{T}_{10}\left(\mathrm{P}_{1} \mathrm{~N}_{3}\right)$ & 211 & 25.60 & 122 & 15.87 & 34 & 4.30 \\
\hline $\mathrm{T}_{11}\left(\mathrm{P}_{1} \mathrm{~N}_{4}\right)$ & 285 & 35.17 & 158 & 20.86 & 63 & 8.07 \\
\hline $\mathrm{T}_{12}\left(\mathrm{P}_{1} \mathrm{~N}_{5}\right)$ & 240 & 29.29 & 139 & 18.25 & 47 & 5.98 \\
\hline $\mathrm{T}_{13}\left(\mathrm{P}_{1} \mathrm{~N}_{6}\right)$ & 276 & 33.85 & 150 & 19.78 & 55 & 7.03 \\
\hline $\mathrm{T}_{14}\left(\mathrm{P}_{1} \mathrm{~N}_{7}\right)$ & 231 & 28.17 & 133 & 17.43 & 43 & 5.47 \\
\hline $\mathrm{T}_{15}\left(\mathrm{P}_{2} \mathrm{~N}_{1}\right)$ & 146 & 18.11 & 88 & 11.80 & 27 & 3.45 \\
\hline $\mathrm{T}_{16}\left(\mathrm{P}_{2} \mathrm{~N}_{2}\right)$ & 134 & 16.61 & 84 & 11.19 & 26 & 3.33 \\
\hline $\mathrm{T}_{17}\left(\mathrm{P}_{2} \mathrm{~N}_{3}\right)$ & 130 & 16.10 & 80 & 10.62 & 24 & 3.07 \\
\hline $\mathrm{T}_{18}\left(\mathrm{P}_{2} \mathrm{~N}_{4}\right)$ & 197 & 25.75 & 109 & 15.23 & 30 & 4.06 \\
\hline $\mathrm{T}_{19}\left(\mathrm{P}_{2} \mathrm{~N}_{5}\right)$ & 171 & 21.65 & 105 & 14.12 & 29 & 3.77 \\
\hline $\mathrm{T}_{20}\left(\mathrm{P}_{2} \mathrm{~N}_{6}\right)$ & 177 & 22.65 & 107 & 14.44 & 29 & 3.80 \\
\hline $\mathrm{T}_{21}\left(\mathrm{P}_{2} \mathrm{~N}_{7}\right)$ & 155 & 19.28 & 91 & 12.25 & 27 & 3.50 \\
\hline $\mathrm{T}_{22}\left(\mathrm{P}_{3} \mathrm{~N}_{1}\right)$ & 107 & 15.06 & 59 & 9.13 & 16 & 2.36 \\
\hline $\mathrm{T}_{23}\left(\mathrm{P}_{3} \mathrm{~N}_{2}\right)$ & 97 & 13.23 & 49 & 7.29 & 14 & 2.03 \\
\hline $\mathrm{T}_{24}\left(\mathrm{P}_{3} \mathrm{~N}_{3}\right)$ & 83 & 10.95 & 46 & 6.81 & 9 & 1.28 \\
\hline $\mathrm{T}_{25}\left(\mathrm{P}_{3} \mathrm{~N}_{4}\right)$ & 126 & 19.48 & 77 & 13.05 & 23 & 3.65 \\
\hline $\mathrm{T}_{26}\left(\mathrm{P}_{3} \mathrm{~N}_{5}\right)$ & 112 & 16.14 & 65 & 10.53 & 20 & 3.1 \\
\hline $\mathrm{T}_{27}\left(\mathrm{P}_{3} \mathrm{~N}_{6}\right)$ & 124 & 18.80 & 70 & 11.57 & 22 & 3.48 \\
\hline $\mathrm{T}_{28}\left(\mathrm{P}_{3} \mathrm{~N}_{7}\right)$ & 111 & 15.95 & 59 & 9.49 & 17 & 2.58 \\
\hline $\operatorname{SEm}( \pm)$ & 2.98 & 0.50 & 2.46 & 0.37 & 2.35 & 0.32 \\
\hline $\operatorname{LSD}(\mathbf{P} \leq 0.05)$ & 8.46 & 1.42 & NS & NS & 6.66 & 0.90 \\
\hline
\end{tabular}

The interaction effect between pruning and nutrient revealed fruit yield was significantly different in Ambe and Hasth bahar, whereas it was statistically at par in Mrig bahar. $\mathrm{T}_{11}$ 
$\left(\mathrm{P}_{1} \mathrm{~N}_{4}\right)$ gave the maximum fruit yield $(35.17$ $\mathrm{kg} /$ plant, $20.86 \mathrm{~kg} /$ plant and $8.07 \mathrm{~kg} / \mathrm{plant}$ ) at Ambe, Mrig and Hasth bahar followed by $\mathrm{T}_{13}$ $\left(\mathrm{P}_{1} \mathrm{~N}_{6}\right)(33.85 \mathrm{~kg} / \mathrm{plant}, 19.78 \mathrm{~kg} / \mathrm{plant}$ and $7.03 \mathrm{~kg} /$ plant), whereas minimum fruit yield was recorded $(8.86 \mathrm{~kg} / \mathrm{plant}, 4.25 \mathrm{~kg} /$ plant and $0.92 \mathrm{~kg} /$ plant $)$ in $\mathrm{T}_{3}\left(\mathrm{P}_{0} \mathrm{~N}_{3}\right)$ at three seasons respectively. It might be because of more open tree canopy with wider leaf area resulted allowing more light penetration that led assimilation more photosynthetic materials which increased the number of laterals, leaf area, number of spurs, flower bud, fruit set and size, thus increasing total yield and also less competition for the growth of individual fruit as compared to unpruned trees (Kumar et al., 2014). NPK in association of biofertilizer, VAM and Vermicompost at desired amount enhanced leaf chlorophyll content resulting in accumulation of more photosynthates, ultimately resulted in higher yield (Yadav et al., 2011 and Kundu et al., 2011). Similar result also found in lemon cv. Pant Lemon-1 (Mishra et al., 2011).

In conclusion, the present results suggest that integrated application of inorganic fertilizers, organic and biological sources of nutrients in an efficient way would not only reduce the sole dependence on inorganic fertilizers but also influence the flowering-fruiting in lemon. Besides, pruning has also significant effect in fruit yield. Among several levels pruning and nutrients application, light pruning $(25 \mathrm{~cm}$ pruning from the terminal portion of the shoot) along with integrated use of fertilizers viz. 75\% RDF +Vermicompost + Azotobacter + Vesicular Arbuscular Mycorrhiza proved as best in terms of quality lemon production for this region.

\section{Acknowledgements}

Authors are grateful to Prof. S. K. Ghosh, Head, Department of Pomology and Post Harvest Technology for providing guidance and financial support during research.

\section{References}

Gomez, K.A. and Gomez, A.A. 1983. Problem data. In: Statistical procedures for Agricultural Research (2 $2^{\text {nd }}$ edition), Wiley-Inter science Publication (John Wiley and Sons); New York, USA, pp 275-315.

Iglesias, D.J., Cercos, M., Colmenero-Flores, J.M., Naranjo, M.A., Rios, G., Carrera, E., Ruiz-Rivero, O., Lliso, I., Morillon, R., Tadeo, F. R., Talon, M. 2007. Physiology of citrus fruiting. Br. J. Plant Physiol., 19(4): 333-362.

Khehra, S. and Bal, J.S. 2014. Influence of organic and inorganic nutrient sources on growth of lemon (Citrus limon Burm.) Cv. Baramasi. J. Exp. Biol. and Agric. Sci., 2(1S): 126-129.

Kumar, H., Katiyar, P.N., Singh, A.K., Rajkumar, B.V. 2014. Effect of different pruning severity on growth and yield of ber (Zizyphus mauritiana Lamk), cv. Banarsi Karaka. Int. J. Curr. Microbiol. and Appl. Sci., 3(5): 935940.

Kundu, S., Datta, P., Mishra, J., Rashmi, K. and Ghosh, B. 2011. Influence of biofertilizer and inorganic fertilizer in pruned mango orchard cv. Amrapali. $J$. Crop and Weed, 7(2): 100-103.

Mishra, N.K., Mishra, K.K., La, R.L., Mishra, D.S. and Goswami, A.K. 2011. Influence of integrated use of organic and inorganic source of nutrient on productivity and quality of lemon (Citrus limon Burm.) cv. Pant Lemon-1 under terai condition of Uttrakhand. The Hort. J., 24(2): 67-69.

Nath, J.C. and Baruah, K. 1999. Regulation of flowering time, plant growth and yield in Assam Lemon (Citrus limon) with the help of pruning and growth regulators. Indian J. Agric. Sci., 69(4): 292-294.

Shaban, A.E.A. and Haseeb, G.M.M. 2009. 
Effect of pruning severity and spraying some chemical substances on growth and fruiting of guava Trees. Am. Eur. J. Agri. and Env. Sci., 5(6): 825-831.

Singh, G. and Dhaliwal, G.S. 2004. Effect of different pruning levels on fruit yield and quality of guava (Psidium guajava L.) cv. Sardar. Haryana J. Hort. Sci., 33(1/2): 83-84.

Singh, J.K., Singh, D.K., Prasad, J. and Singh, H.K. 2009. Studies on integrated nutrient management on flowering behaviour of bael (Aegle mermelos Correa.) cv. Narendra bael.

Singh, R. and Saxena, S.K. 2008. In: Fruits, National Book Trust, India, A-5 Green Park, New Delhi - 110016. pp. 91.

Yadav, A.K., Singh, J.K., Singh, H.K. 2011. Studies on integrated nutrient management in flowering, fruiting, yield and quality of mango cv. Amrapali under high density orcharding. Indian J. Hort., 68(4): 453460.

\section{How to cite this article:}

Ghosh, A., K. Dey, N. Bhowmick, P.S. Medda, A.N. Dey and Ghosh, A. 2017. Reproductive Behaviour of Lemon (Citrus limon Burm.) Affected by Different Pruning Intensities and Integrated Nutrient Management under Various Growing Season. Int.J.Curr.Microbiol.App.Sci. 6(4): 606-614. doi: https://doi.org/10.20546/ijcmas.2017.604.073 